\title{
Baicalein inhibits the migration and invasion of colorectal cancer cells via suppression of the AKT signaling pathway
}

\author{
XIAOJIANG RUI, XI YAN and KAI ZHANG \\ Department of Gastroenterology, 323 Hospital of the People's Liberation Army, Xi'an, Shaanxi 710054, P.R. China
}

Received May 17, 2014; Accepted February 10, 2015

DOI: $10.3892 / \mathrm{ol} .2015 .3935$

\begin{abstract}
The anticancer effect of baicalein has been known for a number of years. However, its anti-metastatic effect and associated mechanisms in colorectal cancer (CRC) remain unclear. The present study investigated the hypothesis that treatment with baicalein may inhibit the proliferation, motility and invasion of human CRC cell lines via regulation of the protein kinase B (AKT) signaling pathway. Baicalein was demonstrated to significantly inhibit the migration and invasion of CRC cells $(\mathrm{P}=0.01)$. Additionally, after treatment with baicalein for $24 \mathrm{~h}$, the protein expression levels of matrix metalloproteinase-2 (MMP-2) and MMP-9 in CRC cells were significantly reduced in a dose-dependent manner $(\mathrm{P}=0.01)$. Furthermore, treatment with baicalein significantly reduced the expression levels of phosphorylated AKT $(\mathrm{P}=0.01)$. In conclusion, baicalein appears to inhibit CRC cell migration and invasion by reducing the expression of MMP-2 and MMP-9 via suppression of the AKT signaling pathway. Thus, baicalein is a potential novel therapeutic agent for patients with CRC.
\end{abstract}

\section{Introduction}

Colorectal cancer (CRC) is a growing threat to public health. It is the second most fatal type of cancer and affects $>1$ million individuals annually worldwide $(1,2)$. Although the removal of primary CRC tumors by surgical resection is typically straightforward, $\leq 50 \%$ of patients with CRC suffer a relapse and eventually succumb to metastatic disease (3), showing that cancer metastasis is the most frequent cause of CRC-associated mortality. Therefore, the development of novel therapeutic agents is required to combat CRC.

Metastasis involves multiple processes, of which degradation of the extracellular matrix (ECM) is an important component (4). Through degradation of the ECM, cancer

Correspondence to: Dr Kai Zhang, Department of Gastroenterology, 323 Hospital of the People's Liberation Army, 6 Jianshe West Road, Xi'an, Shaanxi 710054, P.R. China

E-mail: tougaoyouxiang2014@163.com

Key words: baicalein, colorectal cancer, invasion, AKT cells escape the primary tumor, invade the blood or lymph vessels and migrate to new locations in the body. MMP-2 and MMP-9 are crucial molecules in the degradation of ECM, as well as affecting the metastasis of cancer cells in other ways, such as by inducing angiogenesis (5). In addition, the protein kinase B (AKT) pathway is key in the invasion of cancer cells via the regulation MMPs-2 and -9 (6-8).

Baicalein, which predominantly occurs as an active compound in the root of Scutellaria baicalensis Georgi, is a purified flavonoid with a defined chemical structure (4). Baicalein has been demonstrated to exert antitumor activity in various types of cancer, including CRC (9-11). However, to the best of our knowledge, to date, no studies have been conducted with regard to the anti-metastatic effect of baicalein in CRC. Therefore, the aim of the present study was to explore the effect of baicalein on the invasive ability of $\mathrm{CRC}$, as well as to investigate the underlying mechanisms of this process.

\section{Materials and methods}

Cell culture. The DLD-1 colorectal carcinoma cell line (American Type Culture Collection, Rockville, Maryland, USA) was maintained in RPMI 1640 medium containing 10\% fetal bovine serum (FBS), $100 \mu \mathrm{g} / \mathrm{ml}$ streptomycin and $100 \mathrm{units} / \mathrm{ml}$ penicillin (all from Sigma-Aldrich, St. Louis, MO, USA) in a humidified atmosphere of $5 \% \mathrm{CO}_{2}$ at a temperature of $37^{\circ} \mathrm{C}$.

Cell viability assays. Cell survival was assessed by performing a standard 3-(4,5-dimethylthiazol-2-yl)-2,5-diphenyltetrazolium bromide (MTT) assay, as previously described (4). Briefly, cells were plated in 96-well culture plates at a density of $5 \times 10^{3}$ cells per well and treated with various concentrations of baicalein $(0-120 \mu \mathrm{M})$. After a 24-h incubation, the cells were washed twice with phosphate-buffered saline (PBS) and incubated with $5 \mathrm{mg} / \mathrm{ml}$ MTT (Sigma-Aldrich) for $4 \mathrm{~h}$. The cells were subsequently washed with PBS, solubilized in dimethyl sulfoxide and the optical density of each well was measured using an enzyme-linked immunosorbent assay plate reader (680; Bio-Rad Laboratories, Inc., Hercules, CA, USA) at a wavelength of $490 \mathrm{~nm}$.

Migration and invasion assays. The in vitro migration and invasion assays were conducted in a Transwell ${ }^{\circledR}$ chamber with a Costar ${ }^{\circledR} 8-\mu \mathrm{m}$ pore size polycarbonate membrane (Corning Life Sciences, Cambridge, MA, USA) coated with Matrigel ${ }^{\mathrm{TM}}$ 
or not, as described previously (11). Briefly, cells were trypsinized and suspended to a final concentration of $1 \times 10^{6} \mathrm{cells} / \mathrm{ml}$ in serum-free medium. The suspended cells were treated with varying concentrations of baicalein $(0,10$, 20 and $30 \mu \mathrm{M}$ ) for $24 \mathrm{~h}$ prior to seeding and then added into each upper chamber of the Transwell. The bottom chamber contained medium supplemented with 10\% FBS to serve as a chemoattractant. Following incubation for $24 \mathrm{~h}$ at $37^{\circ} \mathrm{C}$ in a humidified atmosphere of $5 \% \mathrm{CO}_{2}$, all of the non-invaded cells were removed from the upper face of the Transwell membrane using a cotton swab, and the invaded cells were fixed, stained and counted.

Western blot analysis. Western blot analysis was performed as previously described (4). Following treatment with various concentrations of baicalein $(0,10,20$ and $30 \mu \mathrm{M}), 1 \times 10^{6}$ cells were suspended in $100 \mu 1$ lysis buffer $(1 \mathrm{mmol} / 1$ EDTA, $40 \mathrm{mmol} / \mathrm{l} \mathrm{Tris}-\mathrm{HCl}, 150 \mathrm{mmol} / \mathrm{l} \mathrm{KCl}, 100 \mathrm{mmol} / \mathrm{l} \mathrm{NaVO}_{3}$, $1 \%$ Triton X-100 and $1 \mathrm{mmol} / 1$ phenylmethylsulphonyl fluoride; $\mathrm{pH} 7.5)$. The proteins $(60 \mu \mathrm{g})$ were separated by $8 \%$ SDS-polyacrylamide gel electrophoresis and transferred onto polyvinylidene fluoride membranes. The membranes were subsequently blocked with non-fat milk [5\% in Tris-buffered saline and Tween-20 (TBST) buffer] at $37^{\circ} \mathrm{C}$ for $1 \mathrm{~h}$ to prevent non-specific binding. After $1 \mathrm{~h}$, the membranes were incubated overnight with antibodies against polyclonal AKT (cat no. 9272), monoclonal phosphorylated-AKT (p-AKT; cat no. 4060), polyclonal MMP-2 (cat no. 4022), polyclonal MMP-9 (cat no. 3852) or monoclonal $\beta$-actin (cat no. 3700; Cell Signaling Technology, Beverly, MA, USA) in TBST containing 5\% non-fat milk at a temperature of $4^{\circ} \mathrm{C}(12,13)$. The membranes were subsequently incubated with a horseradish peroxidase goat anti-mouse (cat no. 7076) or anti-rabbit IgG (cat no. 7077) antibodies (Cell Signaling Technology) for $1 \mathrm{~h}$ at room temperature. The bands were detected using an enhanced chemiluminescence (ECL) kit (Amersham ECL Plus; GE Healthcare, Freiburg, Germany) and exposed to autoradiography film. Densitometric analysis was performed using Image $\mathbf{J}$ software (National Institutes of Health, Bethesda, MD, USA). The results are expressed as relative expression (\%) compared with the control

Statistical analysis. All statistical analyses were performed using SPSS statistical software (version 16.0; SPSS, Inc., Chicago, IL, USA). Experiments were repeated three times and data were analyzed using Student's t-test. All statistical tests and corresponding P-values were two sided. $\mathrm{P}<0.05$ was considered to indicate a statistically significant difference. Correlation analysis was performed using a Z-test $(13,14)$.

\section{Results}

Baicalein inhibits the proliferation of DLDI cells. The anti-proliferation effects of baicalein at various concentrations $(0-120 \mu \mathrm{M})$ on DLD1 cells are shown in Fig. 1. At a concentration of $50 \mu \mathrm{M}$, baicalein significantly inhibited the proliferation of the DLD1 cells $(\mathrm{P}<0.05)$. However, at concentrations $<50 \mu \mathrm{M}$, the inhibitory effect of baicalein was not significant; thus, a concentration range of baicalein $<50 \mu \mathrm{M}$ was selected for all subsequent experiments.

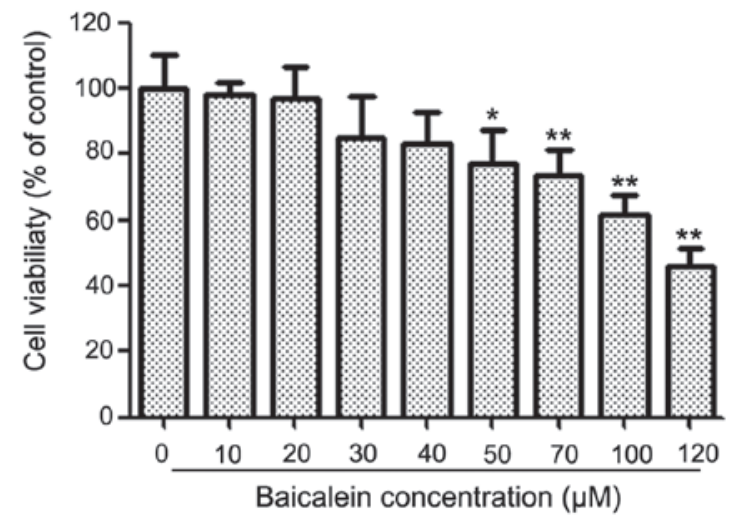

Figure 1. Cellular viability of DLD1 cells. DLD1 cells were treated with increasing concentrations of baicalein and after $24 \mathrm{~h}$ their viability was determined using an 3-(4,5-dimethylthiazol-2-yl)-2,5-diphenyltetrazolium bromide assay. Values represent the mean \pm standard deviation of three independent experiments performed in triplicate. ${ }^{*} \mathrm{P}<0.05$ and ${ }^{* *} \mathrm{P}<0.01$ vs. control group.

Baicalein inhibits the migration and invasion of DLD1 cells. Fig. 2 shows the effect of baicalein on cell migration and invasion in DLD1 cells that were treated with $0,10,20$ and $30 \mu \mathrm{M}$ baicalein for $24 \mathrm{~h}$, respectively. Treatment with baicalein significantly reduced the invasion and migration of DLD1 cells in a concentration-dependent fashion $(\mathrm{P}<0.01)$.

Baicalein suppresses the expression of MMP-2 and MMP-9. The protein expression levels of MMP-2 and MMP-9 in DLD1 cells that were exposed to different concentrations of baicalein was examined. Cells were treated with $0,10,20$ and $30 \mu \mathrm{M}$ baicalein for $24 \mathrm{~h}$ and then subjected to western blotting. Fig. 3A and B shows that baicalein significantly reduced the protein expression levels of MMP-2 and MMP-9 in a concentration-dependent manner compared with the control group $(\mathrm{P}<0.01)$.

Involvement of the AKT signaling pathway in the antimetastatic mechanism of baicalein. The present study also investigated effect of baicalein on the AKT signaling pathway in DLD1 cells. Western blotting indicated that treatment with baicalein significantly reduced the relative expression levels of $\mathrm{p}$-AKT compared with AKT in a concentration-dependent manner $(\mathrm{P}<0.01$; Fig. 4A and $\mathrm{B})$.

\section{Discussion}

Baicalein has been demonstrated to exhibit antitumor effects in various types of cancer $(9,15,16)$. However, the antimetastatic effect and the associated mechanisms in CRC cells remains unclear. The present study revealed that baicalein significantly inhibits the invasive and metastatic ability of CRC cells by regulating the expression levels of MMP-2 and -9 via inhibition of the AKT signaling pathway. To the best of our knowledge, the present study is the first to investigate the antimetastatic effect of baicalein on CRC cells.

A Transwell chamber assay was employed to detect the antimetastatic effect of baicalein on CRC cells. The results demonstrated that treatment with baicalein significantly reduced the migration and invasion of CRC cells. These 

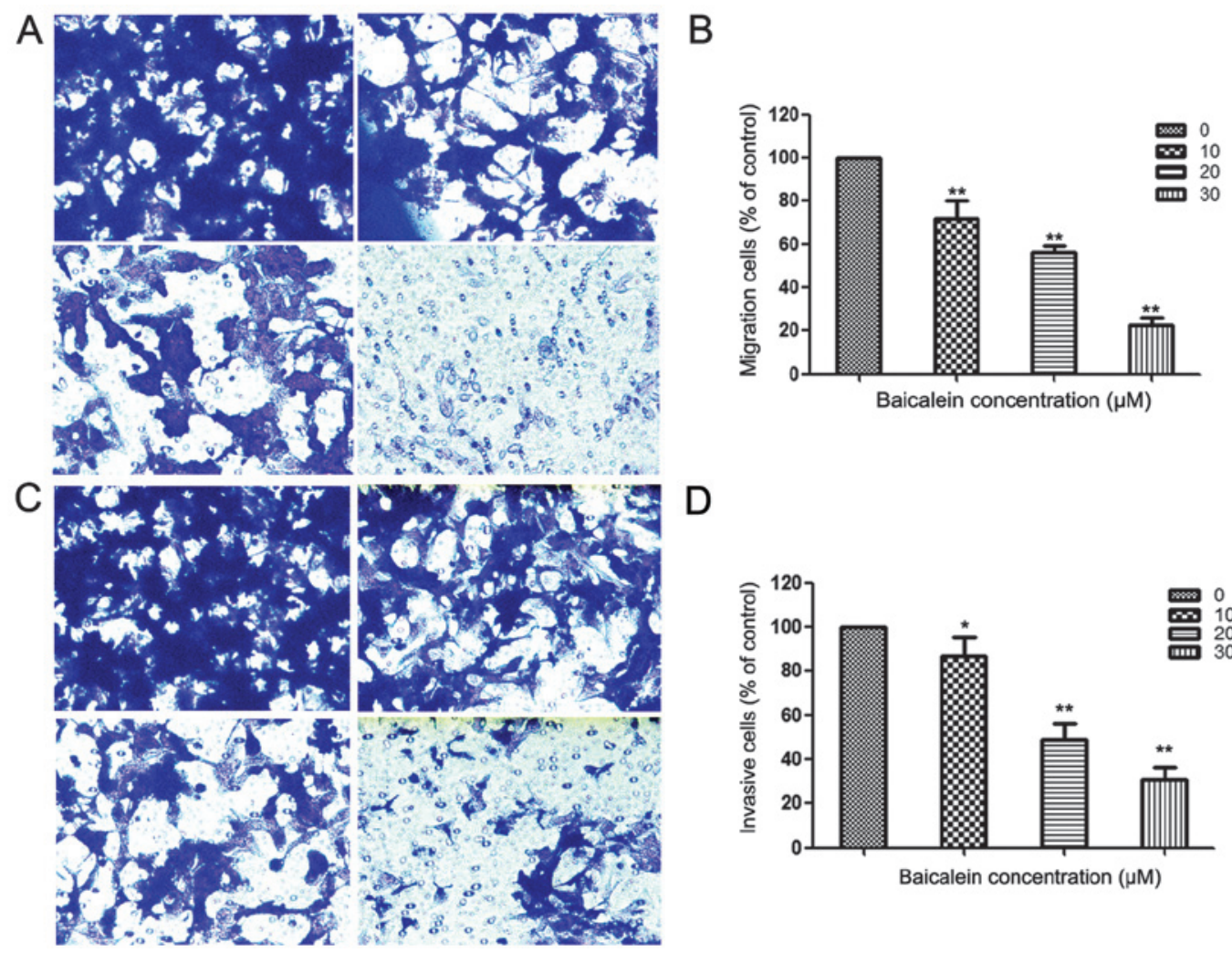

Figure 2. Effect of baicalein treatment on the invasive ability of DLD1 cells. (A) For the migration assay, cells pre-incubated with baicalein $(0,10,20$ and $30 \mu \mathrm{M}$ ) were plated onto the upper wells of the Transwell ${ }^{\boxplus}$ chamber. The spontaneous migration in cells treated with dimethyl sulfoxide (DMSO) was designated as the control. (B) The migration rate (\%) was expressed as a percentage of the control ( $0 \mu \mathrm{M}$ baicalein). (C) For the invasion assay, DLD1 cells pre-incubated with baicalein $(0,10,20$ and $40 \mu \mathrm{M})$ were plated onto the upper wells of the chamber and fetal bovine serum (10\%) was added to the lower wells for $24 \mathrm{~h}$ to induce cell invasion. After $24 \mathrm{~h}$, invaded cells on the bottom side of the filter were fixed, stained and measured. The spontaneous migration in cells treated with DMSO was designated as the control. (D) The invasion rate (\%) was expressed as a percentage of the control (0 $\mu \mathrm{M}$ baicalein). Values represent the mean \pm standard deviation of three independent experiments performed in triplicate. ${ }^{*} \mathrm{P}<0.05$ and ${ }^{* *} \mathrm{P}<0.01$ vs. control group.

A

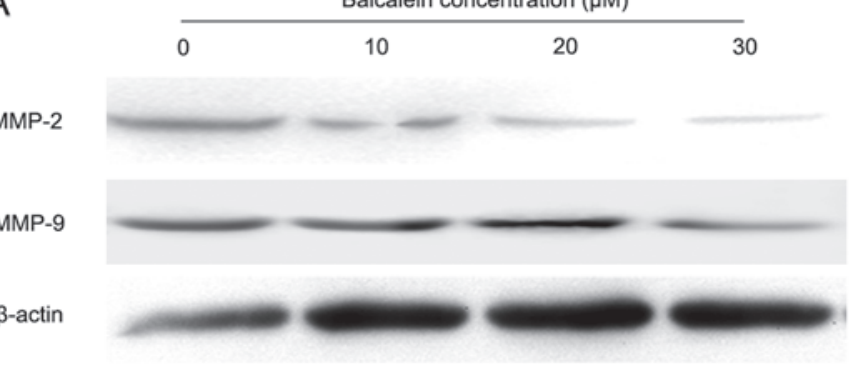

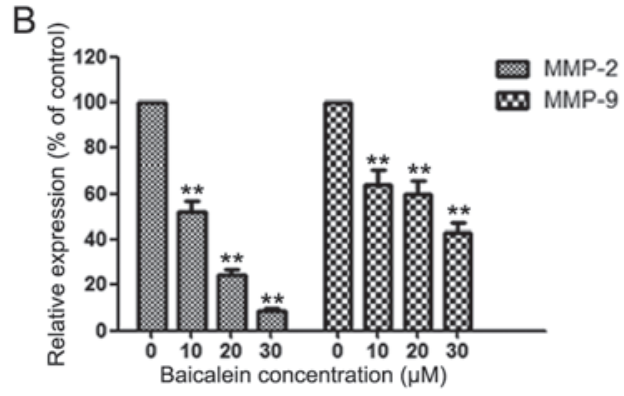

Figure 3. Baicalein treatment suppresses the protein expression levels of MMP-2 and -9 in DLD1 cells. (A) DLD1 cells were treated with baicalein $(0,10,20$ and $30 \mu \mathrm{M}$ ) for $24 \mathrm{~h}$ and then subjected to western blotting to analyze the expression of MMP-2 and -9. (B) Quantification of MMP-2 and -9 expression. Values represent the mean \pm standard deviation of three independent experiments performed in triplicate. ${ }^{*} \mathrm{P}<0.05$ and ${ }^{* *} \mathrm{P}<0.01$ vs. control group. MMP, matrix metalloproteinase.

A

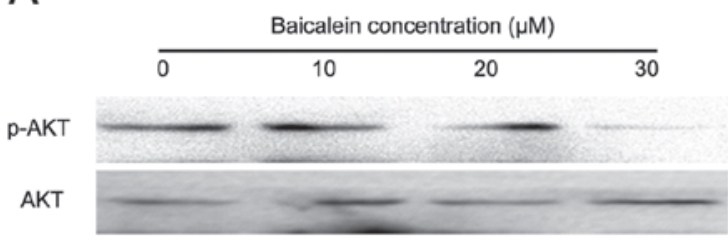

B

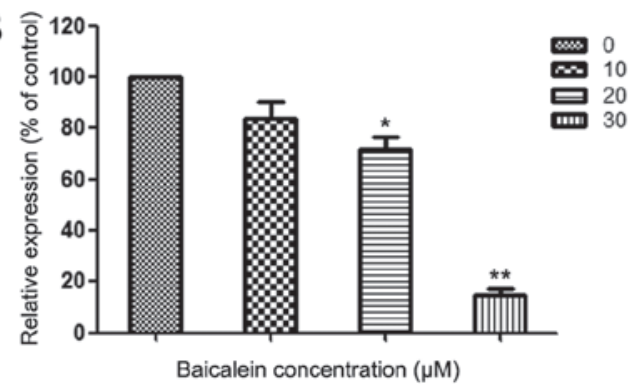

Figure 4. Effect of treatment with increasing concentration of baicalein $(0,10,20$ and $30 \mu \mathrm{M})$ on the AKT signaling pathway. (A) Western blot of the protein expression levels of AKT and p-AKT. (B) Quantified phosphorylation densities of AKT by digital scanning. Values represent the mean \pm standard deviation of three independent experiments performed in triplicate. ${ }^{*} \mathrm{P}<0.05$ and ${ }^{* *} \mathrm{P}<0.01$ vs. control group. AKT, protein kinase B; p-AKT, phosphorylated. 
results are in agreement with previously conducted studies, in which baicalein inhibited the migration and invasion of hepatocellular carcinoma, glioma, breast cancer and tongue cancer cells $(4,11,17,18)$.

Metastasis of cancer cells is a complex multistep process involving cell adhesion, invasion and motility. One of the key steps in metastasis, which involves MMP-2 and -9 , is degradation of the ECM of blood or lymph vessels (19). In CRC patients, plasma MMP-2 levels are correlated with disease stage and survival (19), while MMP-9 expression is correlated with the progression of CRC and hence may be a potential target for the treatment of CRC $(20,21)$. Furthermore, MMP-2 and MMP-9 expression in normal mucosa from patients with CRC are independently associated with the outcome of patients with CRC (22). Therefore, to elucidate the mechanism of the antimetastatic effect of baicalein, the present study determined the expression levels of MMP-2/-9 in CRC cells treated with baicalein, and baicalein was demonstrated to significantly inhibit the protein expression levels of MMP-2/-9 in CRC cells.

Numerous cell signaling pathways are critical in the regulation of MMPs $(4,17,23,24)$. For example, the AKT signaling pathway is associated with the expression of MMP-2/-9 in healthy and cancerous tissues $(24,25)$. Furthermore, the antitumor effects of baicalein treatment are associated with the AKT signaling pathway (26). Hence, interruption of this signaling pathway is one possible approach for the development of novel antimetastatic therapeutic agents. Therefore, the present study investigated the effect of baicalein treatment on the activity of the AKT signaling pathway in CRC cells, and baicalein was shown to significantly reduce the activity of the AKT signaling pathway by inhibiting AKT phosphorylation, subsequently leading to the suppression of CRC cell migration and invasion. However, further investigation is required to determine at what stage the pathway is inhibited.

In conclusion, the present study demonstrated the effect of baicalein treatment on the invasion and metastatic capabilities of CRC cells. Baicalein treatment was demonstrated to significantly correlate with decreased protein expression levels of MMP-2 and MMP-9. Furthermore, the reduced expression of MMP-2 and MMP-9 induced by baicalein was attributed to inhibition of the AKT signaling pathway. This mechanism may contribute to the inhibition of CRC cell invasion and metastasis caused by treatment with baicalein. Thus, the findings of the present study reveal a novel potential therapeutic application of baicalein in antimetastatic CRC therapy. Future studies should focus on determining the anti-metastatic effect of baicalein in vivo.

\section{References}

1. Siegel R, Desantis $C$ and Jemal A: Colorectal cancer statistics. CA Cancer J Clin 64: 104-117, 2014.

2. Unal HU, Demiralay E, Tepeoğlu M, Fidan C and Kilickap S: Lack of relationships between FGF19 staining pattern, lymph node metastasis and locally invasive characteristics of the tumor in colorectal cancers. Asian Pac J Cancer Prev 14: 3151-3154, 2013.

3. de Gramont A: Adjuvant therapy of stage II and III colon cancer. Semin Oncol 32 (Suppl 8): 11-14, 2005.

4. Chen K, Zhang S, Ji Y, et al: Baicalein inhibits the invasion and metastatic capabilities of hepatocellular carcinoma cells via down-regulation of the ERK pathway. PLoS One 8: e72927, 2013.
5. Chaudhary AK, Pandya S, Ghosh K and Nadkarni A: Matrix metalloproteinase and its drug targets therapy in solid and hematological malignancies: an overview. Mutat Res 753: 7-23, 2013.

6. Chung TW, Lee YC and Kim CH: Hepatitis B viral HBx induces matrix metalloproteinase-9 gene expression through activation of ERK and PI-3K/AKT pathways: involvement of invasive potential. FASEB J 18: 1123-1125, 2004.

7. Choo EJ, Rhee YH, Jeong SJ, et al: Anethole exerts antimetatstaic activity via inhibition of matrix metalloproteinase 2/9 and AKT/mitogen-activated kinase/nuclear factor kappa B signaling pathways. Biol Pharm Bull 34: 41-46, 2011.

8. Choi YA, Lim HK, Kim JR, et al: Group IB secretory phospholipase A2 promotes matrix metalloproteinase-2-mediated cell migration via the phosphatidylinositol 3-kinase and Akt pathway. J Biol Chem 279: 36579-36585, 2004.

9. Kim DH, Hossain MA, Kang YJ, et al: Baicalein, an active component of Scutellaria baicalensis Georgi, induces apoptosis in human colon cancer cells and prevents AOM/DSS-induced colon cancer in mice. Int J Oncol 43: 1652-1658, 2013.

10. Kim SJ, Kim HJ, Kim HR, et al: Antitumor actions of baicalein and wogonin in HT-29 human colorectal cancer cells. Mol Med Rep 6: 1443-1449, 2012.

11. Wang L, Ling Y, Chen Y, et al: Flavonoid baicalein suppresses adhesion, migration and invasion of MDA-MB-231 human breast cancer cells. Cancer Lett 297: 42-48, 2010.

12. Yang ZH, Li SN, Liu JX, Guo QX and Sun XW: MMP-9 polymorphisms are related to serum lipids levels but not associated with colorectal cancer susceptibility in Chinese population. Mol Biol Rep 39: 9399-9404, 2012.

13. Chen JS, Wang Q, Fu XH, et al: Involvement of $\mathrm{PI} 3 \mathrm{~K} / \mathrm{PTEN} / \mathrm{AKT} / \mathrm{mTOR}$ pathway in invasion and metastasis in hepatocellular carcinoma: association with MMP-9. Hepatol Res 39: 177-186, 2009.

14. Jung CH, Kim EM, Park JK, et al: Bmal1 suppresses cancer cell invasion by blocking the phosphoinositide 3-kinase-Akt-MMP-2 signaling pathway. Oncol Rep 29: 2109-2113, 2013.

15. Li HL, Zhang S, Wang Y, et al: Baicalein induces apoptosis via a mitochondrial-dependent caspase activation pathway in T24 bladder cancer cells. Mol Med Rep 7: 266-270, 2013.

16. Lin YT, Yang JS, Lin HJ, et al: Baicalein induces apoptosis in SCC-4 human tongue cancer cells via a $\mathrm{Ca}^{2+}$-dependent mitochondrial pathway. In Vivo 21: 1053-1058, 2007.

17. Zhang Z, Lv J, Lei X, et al: Baicalein reduces the invasion of glioma cells via reducing the activity of $\mathrm{p} 38$ signaling pathway. PLoS One 9: e90318, 2014.

18. Zhang Y, Song L, Cai L, Wei R, Hu H and Jin W: Effects of baicalein on apoptosis, cell cycle arrest, migration and invasion of osteosarcoma cells. Food Chem Toxicol 53: 325-333, 2013.

19. Waas ET, Hendriks T, Lomme RM and Wobbes T: Plasma levels of matrix metalloproteinase-2 and tissue inhibitor of metalloproteinase-1 correlate with disease stage and survival in colorectal cancer patients. Dis Colon Rectum 48: 700-710, 2005.

20. Kim EY, Song HY, Kim JC, et al: Mmp-9 expression after metallic stent placement in patients with colorectal cancer: association with in-stent restenosis. Radiology 271: 901-908, 2014.

21. Koskensalo S, Hagström J, Linder N, et al: Lack of MMP-9 expression is a marker for poor prognosis in Dukes' B colorectal cancer. BMC Clin Pathol 12: 24, 2012.

22. Langers AM, Verspaget HW, Hawinkels LJ, et al: MMP-2 and MMP-9 in normal mucosa are independently associated with outcome of colorectal cancer patients. Br J Cancer 106: 1495-1498, 2012.

23. Hsieh $\mathrm{CH}$, Cheng $\mathrm{LH}, \mathrm{Hsu} \mathrm{HH}$, et al: Apicidin-resistant HA22T hepatocellular carcinoma cells strongly activated the Wnt/ $\beta$-catenin signaling pathway and MMP-2 expression via the IGF-IR/PI3K/Akt signaling pathway enhancing cell metastatic effect. Biosci Biotechnol Biochem 77: 2397-2404, 2013.

24. Jin UH, Suh SJ, Chang HW, et al: Tanshinone IIA from Salvia miltiorrhiza BUNGE inhibits human aortic smooth muscle cell migration and MMP-9 activity through AKT signaling pathway. J Cell Biochem 104: 15-26, 2008.

25. Chen JS, Huang XH, Wang Q, et al: Sonic hedgehog signaling pathway induces cell migration and invasion through focal adhesion kinase/AKT signaling-mediated activation of matrix metalloproteinase (MMP)-2 and MMP-9 in liver cancer. Carcinogenesis 34: 10-19, 2013.

26. Zhang HB,Lu P, Guo QY,Zhang ZH and Meng XY: Baicalein induces apoptosis in esophageal squamous cell carcinoma cells through modulation of the PI3K/Akt pathway. Oncol Lett 5: 722-728, 2013. 